\title{
Reprint of Female C57BL/6 mice show consistent individual differences in spontaneous interaction with environmental enrichment that are predicted by neophobia
}

\author{
Michael D. Walker*, Georgia Mason \\ Animal and Poultry Science, University of Guelph, Guelph, Ontario, N1G 2W1, Canada
}

\section{A R T I C L E I N F O}

\section{Article history:}

Received 22 September 2010

Received in revised form 1 June 2011

Accepted 4 June 2011

Available online 4 October 2011

\section{Keywords:}

Mice

Environmental enrichment

Neophobia

Anhedonia

\begin{abstract}
A B S T R A C T
Environmental enrichment typically improves learning, increases cortical thickness and hippocampal neurogenesis, reduces anxiety, and reduces stereotypic behaviour, yet sometimes such effects are absent or even reversed. We investigated whether neophobia governs how mice interact with enrichments, since this could explain why enrichments vary in impact. Female C57BL/6 mice, previously screened for neophobia, had free access to enriched cages connected to their standard cages. The relative consumption of food in each cage revealed approximate dwelling times; the use of two enrichments was also measured. High neophobia significantly predicted reduced use of the enriched cage. Thus even within this homogeneous population, provided with identical enrichments, differential neophobia predicted differential enrichment use.
\end{abstract}

(C) 2011 Elsevier B.V. All rights reserved.
In neuroscience, environmental enrichment refers to "any housing conditions that facilitate enhanced sensory, cognitive and motor stimulation relative to standard housing conditions" [1]. Effective enrichments typically have beneficial effects: enhanced learning and memory; increased cortical thickness and hippocampal neurogenesis; reduced anxiety; reduced drug selfadministration; accelerated recovery from a range of clinical conditions; and/or reduced stereotypic behaviour [1-8]. However, despite this largely positive picture, non-significant or even contradictory findings are often published. Thus environmental enrichment sometimes increases anxiety compared with controlhoused animals [9,10]; has no effect on corticosterone levels [11] or even increases them [9,12]; increases the development of amyloid plaques, in certain Alzheimer's models [1]; and induces stronger drug preferences than in control animals [13]. The causes of this variation have not been investigated. However, it seems likely that the precise nature of the enrichments provided, combined with particular traits of the subjects themselves, together determine the degree to which the enrichments are interacted with and the affective consequences of this interaction. Interaction time is important: direct contact is required for enrichment to have positive effects on,

DOI of original article: 10.1016/j.bbr.2011.06.003.

A publishers' error resulted in this article appearing in the wrong issue. The article is reprinted here for the reader's convenience and for the continuity of the special issue. For citation purposes, please use the original publication details Behavioural Brain Research 224 (2011) 207-212.

* Corresponding author. Tel.: +1 519824 4120x53557.

E-mail address: mwalke04@uoguelph.ca (M.D. Walker). for example, cortical weight [14], and experimentally increasing rodents' interaction times enhances such benefits [15,16]. Affective consequences of interaction are also probably impactful, because stress reduction is a likely mechanism by which enrichments exert their potential benefits $[17,18]$, with increased stress, in contrast, antagonising such effects [19].

One key factor affecting the impact of enrichments is therefore likely to be the exact nature and presentation of the objects supplied. For example, the degree of novelty introduced by moving or replacing enrichments varies greatly between studies [1], and this may differentially affect avoidance of these objects. To illustrate, in one study [20], rats housed in two inter-connected cages, one empty and the other containing novel objects replaced daily, chose to avoid the novel objects, preferring the empty cage. Even apparently subtle differences between enrichments can affect their incentive value to rats and mice; thus when experimentally compared, rodents' show different strengths of preference for different models of running wheel [21] and shelter [22], different sizes of novel objects [23], and different types of bedding or nesting [24]. A second crucial factor influencing the impact of enrichments is likely to be the preferences and temperaments of the experimental subjects themselves. To illustrate, individual variation in the degree to which elderly mice find large enriched cages rewarding (incentive value being assessed via break-points to access these cages), predicts the degree to which such enrichment alleviates their stereotypic behaviour [25]. At the group level, "older animals ... often show little interest in environmental enrichments" [25], for reasons that may include physical incapacity, neophobia and anhedonia; while strains varying in neophobia should diverge 
in the extent to which they find novelty stressful, this helping to explain why "strain differences ... [can] alter the responsiveness of the animals to the enrichment paradigm" [1]. Rodents with anhedonia, as can be induced by long-term impoverished caging [26] and also varies with strain [27] may also fail to show much interaction with enrichments that to other rodents act as valued incentives. Overall, variation between rodents in neophobia and anhedonia may thus help explain why not just strain, but also sex, age and individual characteristics can influence experimental outcomes, even when enrichments are carefully standardized [28-35]. Here, we therefore used individual variation in responses to standardized enrichments to conduct preliminary tests of the hypotheses that neophobia and anhedonia affect the extent to which mice interact with environmental enrichments.

Subjects were female C57BL/6J mice from Charles River, age 5 months at the start of the study; females were used to minimize risks of competitive aggression [1,36]. They were maintained on a $12 \mathrm{~h}$ semi-reversed light cycle (lights out at 13:30), at a room temperature of $21 \pm 1{ }^{\circ} \mathrm{C}$ and $48 \%$ relative humidity. Each mouse was pair-housed with a sister (one mouse per pair having a patch shaved for identification), in standard clear polysulfone plastic 'shoebox' cages $(12 \mathrm{H} \mathrm{cm} \times 27 \mathrm{Lcm} \times 16 \mathrm{Wcm})$, containing corn cob bedding, Shepherd Shack Enviro-dry ${ }^{\circledR}$ nesting material, a UDEL polysulfone plastic house, and access to ad lib food and water. The initial pool of animals comprised 31 sibling pairs, from 31 litters. These were all tested for neophobia. Tests were conducted in their home cages: free exposure to novelty is most likely to reveal trait anxiety [cf. forced exposure which affects state; [37], and this approach also avoided non-systematic error from handling, isolation or transport stress. Tests involved inserting a novel object through the cage lid, in the cage corner, down to the bedding. This was conducted $2 \mathrm{~h}$ into the dark period, on two consecutive days, using two different objects: on the first day, a $14 \mathrm{~cm} \times 2.5 \mathrm{~cm}$ strip of tinfoil, and on the second, a plastic drinking straw. After an object had been used once, it was discarded, each cage always being tested with a new item. The latency for each mouse to make first contact was recorded. Each object was left in the cage for $20 \mathrm{~min}$; any mouse making no contact at all was given the maximum score (1200s).

Neophobic responses were used to select 19 pairs for further study, 19 being the number of large enriched cages available for the hypothesis-testing phase (see below). Cagemates' values for each trial were averaged; each pair's latency to touch the foil was regressed against its latency to touch the straw; the line of best fit plotted; and the residual from this line calculated for each pair (reflecting the degree to which its values diverged between both trials). Pairs were ordered by residual value, and the 19 pairs that had the smallest residuals were selected for further study, provided that on further inspection of the data, the two mice in each cage also did not greatly diverge in their neophobic responses (this was judged subjectively). These 19 pairs thus represented the cages from which our two tests generated the most internally consistent data, suggesting the most reliable reflection of trait neophobia.

Sucrose consumption was then used to assess anhedonia in these 19 pairs, following the solid sucrose protocol of Brennan et al. [38], with the modification that mice were not food- or waterdeprived. A small dish containing two pre-weighed sugar cubes was placed in each cage for $30 \mathrm{~min}$, after which it was removed and re-weighed. This was repeated for 12 consecutive days. About half the pairs consumed sugar on the first test day $(n=10)$; the rest took one or more days to commence ingestion $(n=9)$. This yielded an opportunistic additional measure of neophobia (hyponeophagia: $39)$. For the last 6 days most $(n=14)$ pairs were regularly consuming sugar; data from these last 6 days were therefore used to score hedonia (via average daily weight ingested).

Each cage was then connected to a large enriched cage $(26 \mathrm{Hcm} \times 60 L \mathrm{~cm} \times 60 \mathrm{Wcm})$ via a short piece of PVC tubing allowing free access. The enrichment paradigm modelled previous studies where enrichment involves extra space, stable increased physical complexity and opportunities to perform rewarding behaviours (e.g. wheel-running, gnawing), along with a variable component whereby novel objects are regularly changed and rotated $[36,40,41]$. The one difference from these previous studies was that our subjects had a free choice as to whether to enter the enriched cage, a choice that was offered round-the-clock for a full 4 weeks.

All enriched cages were identically provisioned with diverse objects, some remaining there for the entire 4 weeks (plastic drinking straws, a plastic spoon, a cardboard planting pot, an upturned plastic bowl, toothpicks, an old $26 \mathrm{Hcm} \times 18 \mathrm{Lcm} \times 18 \mathrm{Wcm}$ mesh bottom metal rat cage, a surgeon's mask suspended to form a 'hammock', a large sheet of fleece suspended from the cage lid to provide cover, a roll of twine suspended outside the cage with the end hanging into the roof of the cage, and a running wheel); others, present for only one of the 4 weeks (a ball of newspaper, a $2.5 \mathrm{Hcm} \times 15 \mathrm{Lcm} \times 5 W \mathrm{~cm}$ block of wood, plastic vegetables, popsicle sticks, a balloon, plastic container); and yet others still renewed once every 7 days (shredded napkins, cotton balls). Changes to the enrichments and cage layout occurred weekly at cage cleaning, following the protocols of prior studies $[35,39,40]$; on this day, mice were denied access to the enriched cage. Enriched cages were always re-provisioned in the same way so that all 19 remained identical. The enriched cages also contained $a d$ lib food and water. The use of these cages was monitored every $24 \mathrm{~h}$, for the 6 days each week that the mice had access, using three measures of resource consumption. The food present in both the home and enriched cages was weighed daily to calculate how much had been eaten from each. (These values were also statistically compared to the relative surfaces of exposed food in the hoppers in the two cagetypes [60:40], to assess whether food consumption in the enriched cage greater or less than expected by chance.) Consumption of two enrichments was also recorded every $24 \mathrm{~h}$ : the cardboard planter pot, which the mice gradually destroyed through chewing and scratching, was weighed, and the length of string pulled into the cage was measured. Finally, the mice themselves were weighed at the end of the experiment.

All statistical analyses were conducted using Minitab ${ }^{\mathrm{TM}} 15$, data being transformed when necessary to meet assumptions of parametric statistics. To assess day-to-day consistency in behaviour, we ran two-tailed split-half analyses [44], regressing the average values for even days of data collection for each pair against the average values for odd days. In contrast one-tailed tests were used for all analyses pertaining to the hypotheses under test, because predictions were directional [e.g. 42,43]. To create a single neophobia score from each pair to use in analyses of enriched cage use, the two values from the paired cagemates were averaged into a cage score for each trial, and these scores were averaged again to produce a single neophobia score. Cage (equivalent to 'pair') was thus the unit of replication in all analyses, partly because cagemates' activities could not be distinguished in the enriched cages, but primarily because related cagemates are behaviourally very similar [25], and so to avoid statistical pseudoreplication, should not be treated as non-independent [45]. Note that using cage averages is also statistically identical to including individual 'Mouse ID' in the models but treating it as a random effect nested within cage.

Average latencies in the two novel object tests varied greatly between pairs, from just $32.4 \mathrm{~s}$ to 1200 s (i.e. never touching the object in $20 \mathrm{~min}$, as occurred in 5 pairs). For the 19 pairs selected for anhedonia and enrichment phases, pair mean approach times correlated between the two neophobia trials $\left(F_{1,17}=202.5, p<0.001\right)$. The average score in both trials for each mouse also strongly correlated between cagemates $\left(F_{1,17}=11.7, p=0.003\right)$. Latencies to first consume sugar in the sucrose consumption test (hyponeophagia) 


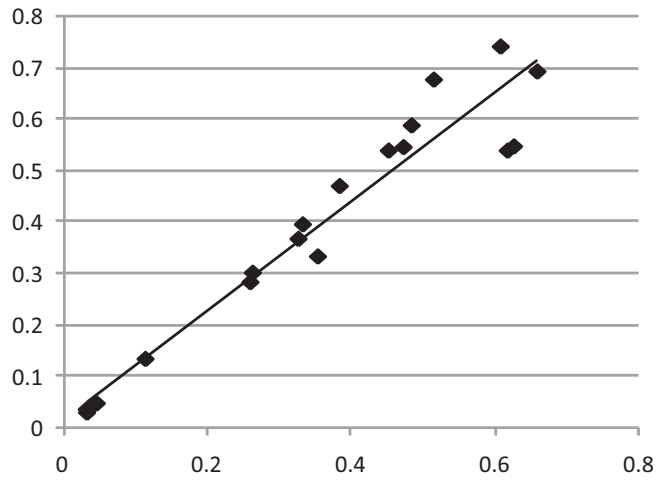

Mean Proportion of Food eaten in the EE Cage on Odd Numbered Days

Fig. 1. Stability of enrichment use as indexed by the proportion of food consumed in the enriched cage on even and odd days. Untransformed data shown here.

were significantly related to novel object scores: long latency pairs in the sucrose test showed longer times to approach the two novel objects $\left(F_{1,15}=4.56, p=0.05\right)$. Once consuming sugar, all pairs also behaved stably and consistently in over the final 6 days of this test, as evidenced by the split-half analysis comparing odd and even days $\left(F_{1,12}=57.66, p<0.001\right)$. Their sucrose consumption also ranged from an average of $0.152 \mathrm{~g} /$ day to $0.348 \mathrm{~g} /$ day (excluding pairs that ate no sugar at all). Novel object scores did not correlate with the anhedonia scores, suggesting these are independent traits $\left(F_{1,12}=0.07, p=0.795\right)$; body weight also did not predict amount of sugar eaten, suggesting this behaviour was driven by hedonia rather than calorific need $\left(F_{1,12}=0.84, p=0.378\right)$.

The use of the enriched cage similarly showed substantial variation between pairs, along with great within-pair day-today consistency. Thus the mean proportion of food ( \pm standard error) eaten from the enriched cage ranged from $0.031 \pm 0.002$ to $0.699 \pm 0.012$, but each caged pair behaved consistently over the 4 weeks of the experiment, as shown by split-half analysis $\left(F_{1,17}=590.08, p<0.001\right.$, Fig. 1$)$. String use varied between a daily mean of $0 \mathrm{~cm}$ a day $( \pm 0)$, and $452.28 \mathrm{~cm} /$ day $( \pm 28.43)$, and each cage's daily string use scores were significantly consistent over time (split-half analysis: $F_{1,17}=31.32, p<0.001$; Fig. $2 \mathrm{~A}$ ). Likewise, pot destruction varied from a daily mean of $0.02 \mathrm{~g}( \pm 0.002)$, to a mean of $0.504 \mathrm{~g}( \pm 0.015)$, and again each cage's daily use scores were significantly consistent over time (split-half analysis: $F_{1,17}=120.71, p<0.001$; Fig. $2 \mathrm{~B}$ ). The proportion of food eaten in the enriched cage positively correlated with string use $\left(F_{1,17}=4.59\right.$, $p=0.047$, Fig. 3A) and with pot consumption $\left(F_{1,17}=21.09\right.$, $p<0.001$, Fig. 3B). The usage of these two enrichments did not covary however $\left(F_{1,17}=1.96, p=0.179\right)$, suggesting that different pairs differentially favour different enrichment items.

Use of the enriched cage, as inferred from food ingestion there, revealed two more findings of note. First, food consumption from the enriched cage showed a cyclical pattern (see Fig. 4); and average values for the first day of each week, immediately after cleaning and enrichment-change, proved significantly lower than average values for the other 5 days of the week (paired $t_{19}=4.76, p<0.001$ ). Second, preference for the enriched cage over the standard cage, as assessed from relative food consumption statistically compared to chance (the relative areas of exposed food in the two pellet hoppers, 40:60) and omitting the four weekly 'disturbance days' where mice reduced their use of the enrichments, varied between pairs: two ate more from the enriched cages than expected by chance $(|t| \geq 2.06$, $p \leq 0.05$ ), while 11 pairs ate there significantly less than expected by chance $(|t| \geq 2.18, p \leq 0.05)$.

Use of the two individually measured enrichments was not predicted by prior scores in the novel object test, nor in the sucrose consumption test (string: $F_{1,17}=0.04, p=0.852$ and $F_{1,12}=0.01$., 0.923 respectively; pot: $F_{1,17}=0.72, p=0.409$ and $F_{1,12}=0.11$, $p=0.747$ respectively). However, novel object test scores did significantly predict the proportion of food eaten in the enriched cage: mice that had taken less time to approach the novel object, when tested in their standard cage, then ate more of their food from the enriched cage $\left(F_{1,17}=3.76, p=0.0345\right.$, Fig. 5$)$. Similarly, mice that had eaten the sugar on the first day it was offered in the sucrose consumption test went on to eat a significantly higher proportion of their food from the enriched cage $\left(T_{16}=2.21, p=0.021\right.$, Fig. 6$)$. Sugar consumption, our index of hedonia, in contrast did not predict the proportion of food that mice ate in the enriched cage $\left(F_{1,12}=1.17\right.$, $p=0.15$ ).

In summary, the present study demonstrates for the first time that there are stable individual differences in voluntary enrichment-use, and that mice that are more fearful of novelty will utilize enrichments less than their bolder conspecifics. Thus the pairs showed large and stable differences in enrichment-use, as quantified via string use, cardboard pot destruction and feeding in the enriched cage. A few subjects appeared to actively prefer the enriched environment (two pairs ate from the enriched cages more than expected by chance), while others found it relatively
A

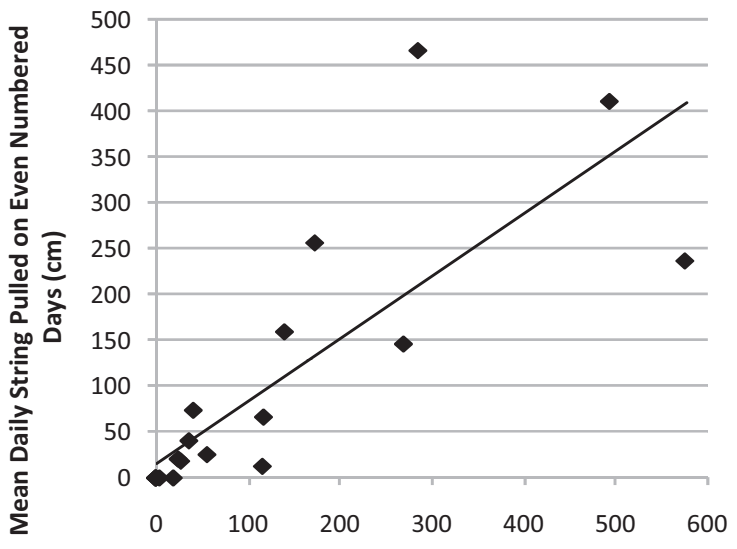

Mean Daily String Pulled on Odd Numbered Days (cm)

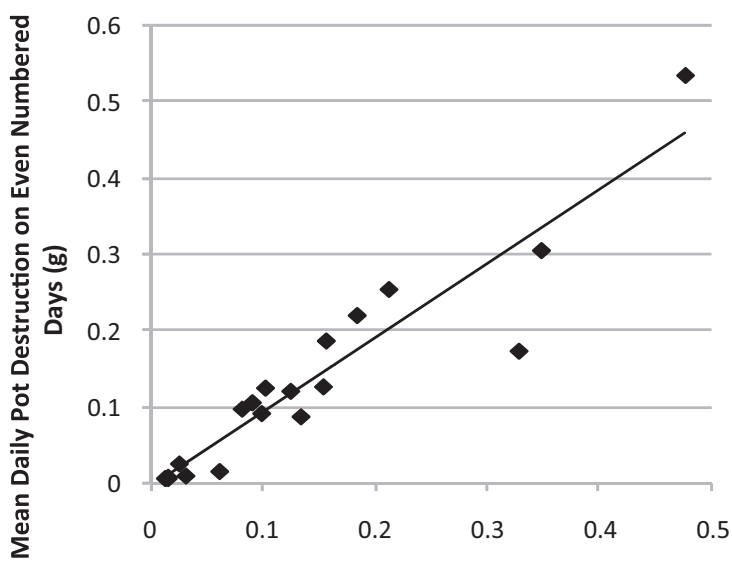

Mean Daily Pot Destruction on Odd Numbered Days (g)

Fig. 2. Stability of enrichment use as indexed by mean daily amount of string pulled (A) and amount of pot destruction (B) of each cage on even and odd days. 

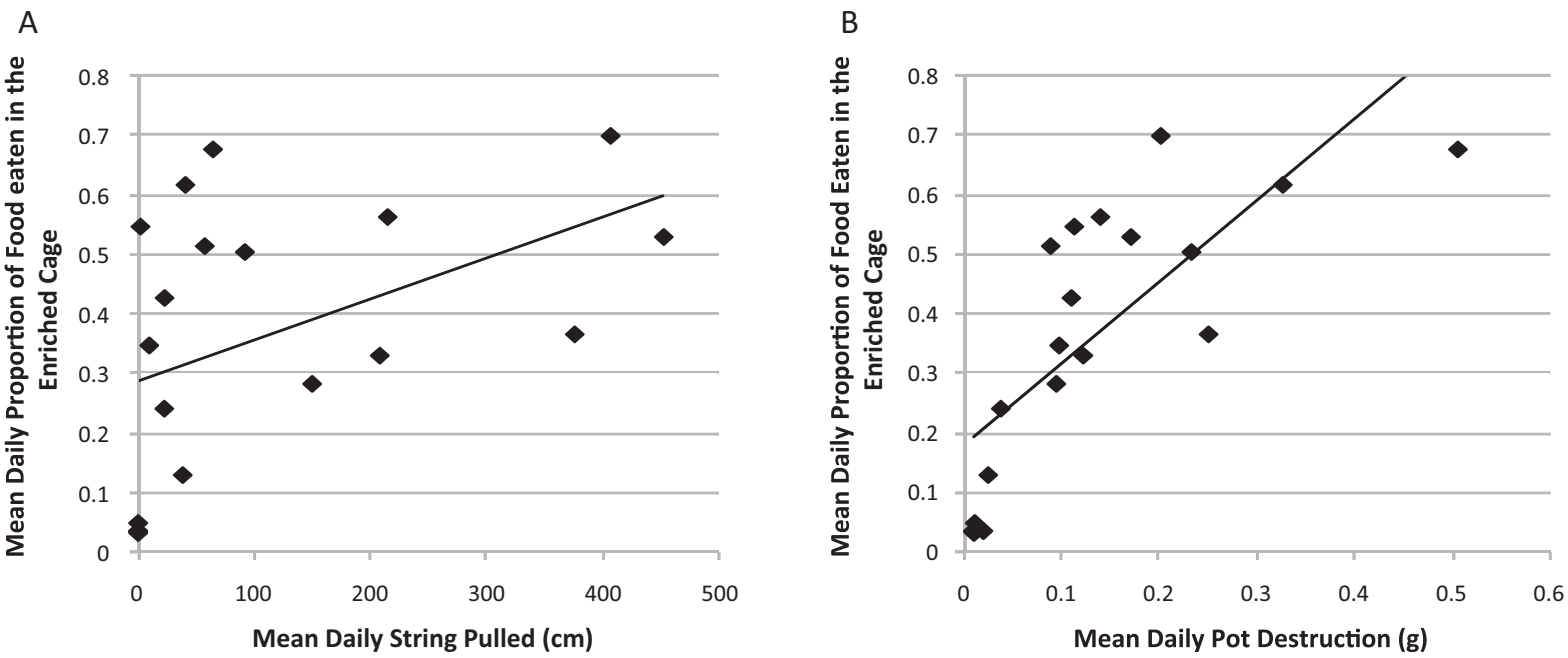

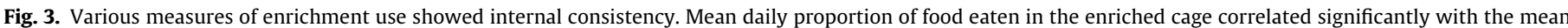
daily string pulled (A) and the mean daily amount of pot destruction (B). Untransformed data shown here.

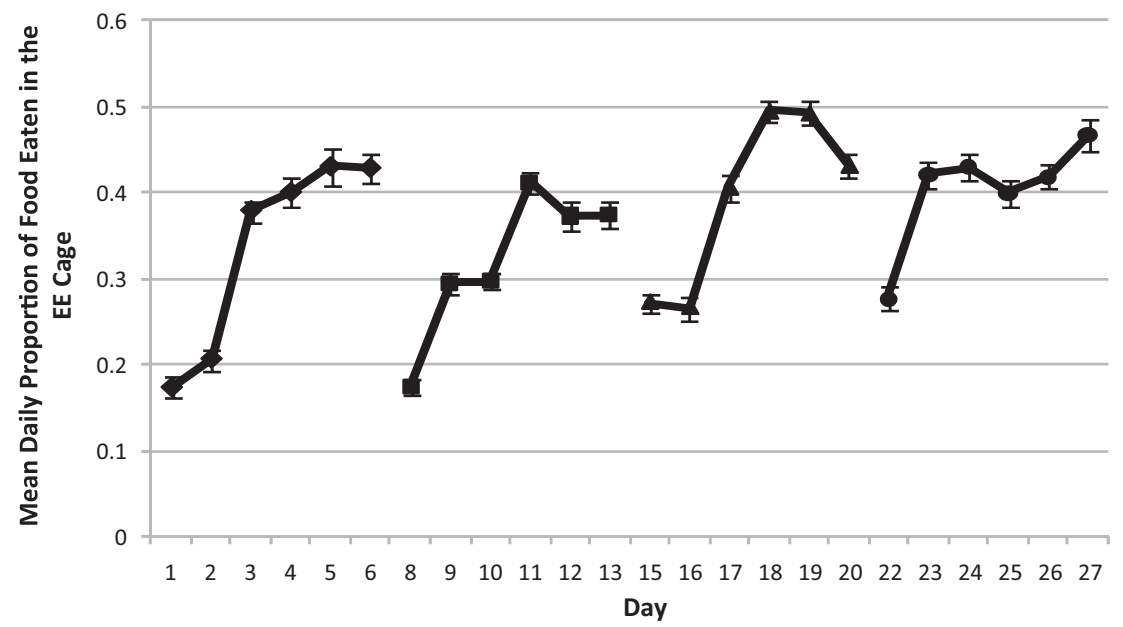

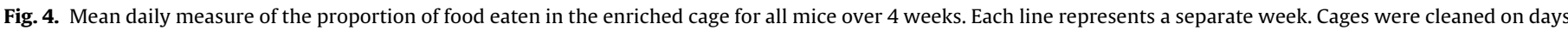

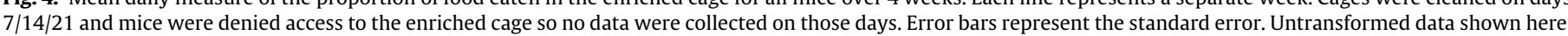

aversive, many pairs eating from the enriched cage less than would be expected by chance. This relatively low overall preference was at odds with a recent experiment from our lab [46] using mice of the same age, sex and source, in which all mice housed in two

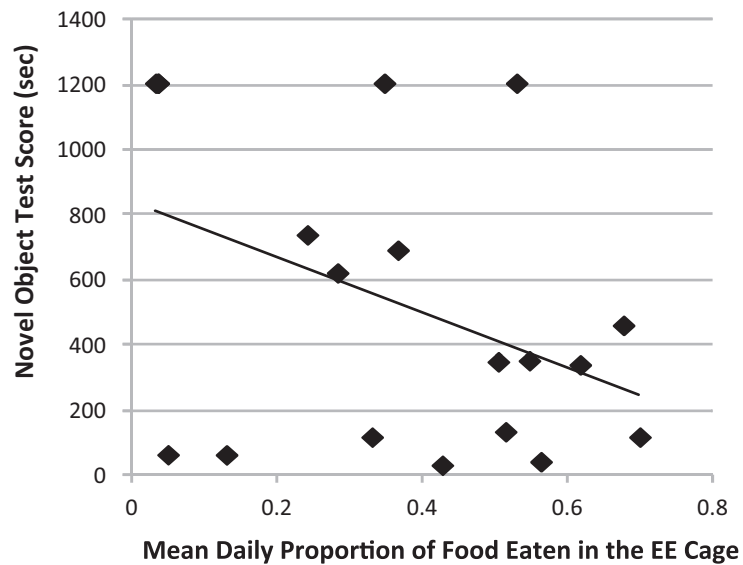

Fig. 5. Significant negative correlation between the score in the novel object test and the proportion of food consumed in the enriched cage. Untransformed data shown here. interconnected standard cages, one containing a regularly renewed Kleenex, a running wheel, and a wooden stick smeared with peanut butter, spent the bulk of their time in the enriched rather than standard cage. The lack of food treats in the current study may be one reason, but another likely reason is the regular rotation and

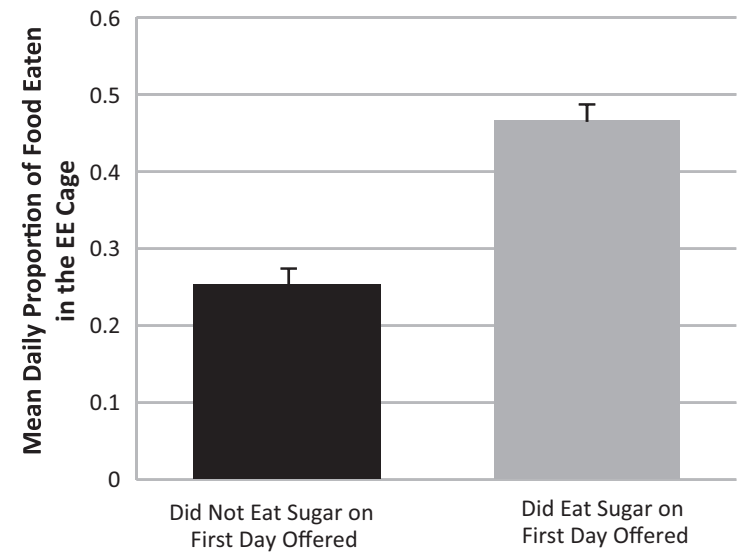

Fig. 6. Mice that ate sugar on the first day that it was offered in the sugar consumption test went on to consume a significantly higher proportion of food from the enriched cage. Untransformed data shown here. 
renewal of novel objects in this experiment-a characteristic of many enrichment paradigms in common use.

Patterns of use certainly suggested that the degree of novelty, and how the mice evaluated this, was an important determinant of enrichment-interaction: over the entire course of the experiment, there was a weekly day-long decrease in enrichmentuse, as indexed by relative feed consumption, after each day of cage-cleaning (standard and enriched cages) and enrichment rotation (enriched cages only). Furthermore, the aspects of phenotype helping to determine enrichment-use also indicated this too: empirically, the most neophobic subjects-as characterised during both novel object tests and hyponeophagia tests-used the enriched cage least. Future work should now replicate and build on this finding, obtaining utilisation scores for more than just two of the many enrichments on offer (e.g. using running wheels with counters); collecting stronger data on overall dwelling times via e.g. distribution of faecal pellets [47] or $24 \mathrm{~h}$ video recordings [48] and quantifying the incentive value of the enriched environments via break point to access [25]. In addition, scoring the mice in a novel environment exploration test would allow for a more robust measure of neophobia when combined with the tests performed here. Anhedonia, in contrast, had no such effects in this study, suggesting either that hedonia was not a key determinant of enrichment-use in this experiment, or that this aspect of the study now needs replicating with a larger sample size (our statistical power was low for this test because five pairs failed to eat any sugar at all), perhaps using a more conventional drinking-bottle-based test less likely to cause hyponeophagia.

Differences in neophobia affecting enrichment use were evident despite using a population that was homogeneous in age, sex and genetics, and despite using highly standardized enrichment and control cages. Differences in neophobia and thence interaction with enrichments are likely to be much larger across studies using diverse mice or a variety of different forms of enrichment. We therefore suggest that variation in enrichment-neophobia could help explain the disparity of findings from studies that use different enrichments and/or mice varying in age, sex or strain. Neophobia could have affected the outcome of past enrichment experiments in two ways. As our data show, it could modulate the subjects' interaction time with the enrichment objects. In addition, it could influence the affective consequences of enrichment exposure; neophobic subjects exposed to novelty-based enrichments may even react with stress responses which could then reduce or even reverse the normally beneficial effects of enrichments. These plausible new explanations for the variable impact of enrichment now need direct test.

Several previous authors have noted that 'enrichment' is a rather loosely defined term, often used to mean any changes made to a standard environment [1,5,49]; and some have suggesting standardizing enrichments as a way to generate more robust, replicable results [e.g. 49]. Our findings concur with this suggestion, in that enrichments differing in tendencies to elicit neophobic responses (for instance as determined by how often they are changed: a variable typically not standardized between experiments) will vary in their impact on the subjects. However, neophobia is not just sensitive to the degree of novelty in an environment: it is a trait that varies with family, age, strain and sex. Therefore, if enrichments fail to have their expected benefits, even when carefully chosen and standardized [cf. 49], neophobia and low enrichment-use are testable potential explanations.

\section{Acknowledgements}

We would like to thank our animal technician Michelle Waechter for caring for the animals used in this study. GJM would also like to thank NSERC for a Discovery grant that helped fund this work.

\section{References}

[1] Nithianantharajah J, Hannan AJ. Enriched environments, experiencedependent plasticity and disorders of the nervous system. Nat Rev Neurosci 2006;7(9):697-709.

[2] Hannan AJ. Environmental enrichment and gene-environment interactions in mouse models of brain disorders. Neuromethods 2010;44:201-16.

[3] Lewis M, Presti M, Lewis J, Turner C. The neurobiology of stereotypy 1: environmental complexity. In: Mason G, Rushen J, editors. Stereotypic animal behaviour. 2nd ed. 2006. p. 190-227.

[4] Mason G, Clubb R, Latham N, Vickery S. Why and how should we use environmental enrichment to tackle stereotypic behaviour? Appl Anim Behav Sci 2007; 102:163-88

[5] Olsson IAS, Dahlborn K. Improving housing conditions for laboratory mice: a review of 'environmental enrichment'. Lab Anim 2002;36:243-70.

[6] van Praag H, Kempermann G, Gage FH. Neural consequences of environmental enrichment. Nat Rev Neurosci 2000;1:191-8.

[7] Smith AL, Corrow DJ. Modifications to husbandry and housing conditions of laboratory rodents for improved well-being. ILAR J 2005;46(2):140-7.

[8] Stairs DJ, Bardo MT. Neurobehavioral effects of environmental enrichment and drug abuse vulnerability. Pharmacol Biochem Behav 2009;92(3):377-82.

[9] Fuss J, Ben A, Nada M-B, Voqt MA, Tuoma C, Pacifici PG, et al. Voluntary exercise induces anxiety-like behaviour in adult C56BL/6J mice correlating with hippocampal neurogenesis. Hippocampus 2010;20(3):364-76.

[10] Van de Weerd HA, Baumans V, Koolhaas JM, Van Zutphen LFM. Strain specific behavioural response to environmental enrichment in the mouse. J Exp Anim Sci 1994;36:117-27.

[11] Tsai PP, Stelzer HD, Hedrich HJ, Hackbarth H. Are the effects of different enrichment designs on the physiology and behaviour of DBA/2 mice consistent? Lab Anim 2003;37:314-27.

[12] Moncek F, Duncko R, Johansson BB, Jezova D. Effect of environmental enrichment on stress related systems in rats. J Neuroendocrinol 2004;16: 423-31.

[13] Bowling SL, Bardo MT. Locomotor and rewarding effects of amphetamine in enriched, social, and isolate reared rats. Pharmacol Biochem Behav 1994;48(2):459-64.

[14] Ferchmin PA, Bennet EL, Rosenzweig MR. Direct contact with enriched environment is required to alter cerebral weights in rats. J Comp Physiol Psychol 1975;88:360-7.

[15] Rosenzweig M, Bennet EL. Cerebral changes in rats exposed individually to an enriched environment. J Comp Physiol Psychol 1972;80(2):304-13.

[16] Kuenzle CC, Knusel A. Mass training of rats in a super enriched environment Physiol Behav 1974;13(2):205-10.

[17] Grote HE, Bull ND, Howard ML, van Dellen A, Blakemore C, Bartlett PF, et al. Cognitive disorders and neurogenesis deficits in Huntington's disease mice are rescued by fluoxetine. Eur J Neurosci 2005;22(8):2081-8.

[18] Arunrut T, Alejandre H, Chen M, Cha J, Russo-Neustadt A. Differential behavioural and neurochemical effects of exercise, reboxetine and citalopram with the forced swim test. Life Sci 2009;84(17-18):584-9.

[19] Ekstrand J, Hellsten J, Tingstrom A. Environmental enrichment, exercise and corticosterone affect endothelial cell proliferation in adult rat hippocampus and prefrontal cortex. Neurosci Lett 2008;442(3):203-7.

[20] Will B, Pallaud B, Ungerer A, Ropartz P. Effects of rearing in different environments on subsequent environmental preference in rats. Dev Psychobio 1979;12(2):151-60.

[21] Banjanin S, Mrosovsky N. Preferences of mice, Mus musculus, for different types of running wheel. Lab Anim 2000;34(3):313-8.

[22] Van Loo PLR, Blom HJM, Meijer MK, Baumans V. Assessment of the use of two commercially available environmental enrichments by laboratory mice by preference testing. Lab Anim 2005;39:58-67.

[23] Williams CM, Riddell PM, Scott LA. Comparisons of preferences for object properties in the rat using paired- and free-choice paradigms. Appl Anim Behav Sci 2008;112(1-2):146-57.

[24] Ras T, van de Ven M, Patterson-Kane EG, Nelson K. Rats' preference for corn versus wood-based bedding and nesting materials. Lab Anim 2002;36(4):420-5.

[25] Tilly S, Dallaire J, Mason G. Middle-aged mice with enrichment-resistant stereotypic behaviour show reduced motivation for enrichment. Anim Behav 2010;80:363-73.

[26] Saenz J, Villagra O, Trias J. Factor analysis of Forced Swimming test, Sucrose Preference test, and open field test on enriched, social and isolation reared rats. Behav Brain Res 2006;169(1):57-65.

[27] Griffiths J, Shanks N, Anisman H. Strain-specific alterations in consumption of a palatable diet following repeated stressor exposure. Pharm Biochem Behav 1992;42(2):219-27.

[28] Cooper JJ, Odberg F, Nicol CJ. Limitations on the effectiveness of environmenta improvement in reducing stereotypic behaviour in bank voles (Clethrionomys glareolus). Appl Anim Behav Sci 1996;48:237-48.

[29] Hadley C, Hadley B, Ephraim S, Yang M, Lewis MH. Spontaneous stereotypy and environmental enrichment in deer mice (Peromyscus maniculatus): reversibility of experience. Appl Anim Behav Sci 2006;97:312-22. 
[30] Harburger L, Lambert T], Frick KM. Age-dependent effects of environmental enrichment on spatial reference memory in male mice. Behav Brain Res 2007; $185: 43-8$.

[31] Kemppinen N, Hau J, Meller A, Mauranen K, Kohila T, Nevalainen T. Impact of aspen furniture and restricted feeding on activity, blood pressure, heart rate, faecal corticosterone and immunoglobulin A excretion in rats (Rattus norvegicus) housed in individually ventilated cages. Lab Anim 2010;44:104-12.

[32] Mirochnic S, Wolf S, Staufenbiel M, Kempermann G. Age effects on the regulation of adult hippocampal neurogenesis by physical activity and environmental enrichment in the APP23 mouse model of Alzheimer disease. Hippocampus 2009;19:1008-18.

[33] Saucier DM, Yager JY, Armstrong EA. Housing environment and sex affect behavioral recovery from ischemic brain damage. Behav Brain Res 2010;214:48-54.

[34] Stam NC, Nithiananthrarajah J, Howard ML, Atkin JD, Cheema SS, Hannan AJ. Sex-specific behavioural effects of environmental enrichment in a transgenic mouse model of amyotrophic lateral sclerosis. Eur J Neurosci 2008;28: 717-23.

[35] Zajac MS, Pang TYC, Wong N, Weinrich B, Leang LSK, Craig JM, et al. Wheel running and environmental enrichment differentially modify exon-specific BDNF expression in the hippocampus of wild-type and pre-motor symptomatic male and female Huntington's disease mice. Hippocampus 2010;20:621-36.

[36] Van Loo PLP, Kruitwagen CLJJ, Van de Weerd HA, Van Zutphen LFM, Baumans $\mathrm{V}$. Influence of cage enrichment on aggressive behaviour and physiological parameters in male mice. Appl Anim Behav Sci 2002;76:65-81.

[37] Treit D. Animal models for the study of anti-anxiety agents: a review. Neurosci Biobehav Rev 1985;9:401-5.

[38] Brennan K, Roberts DCS, Anisman H, Merali Z. Individual differences in sucrose consumption in the rat: motivational and neurochemical correlates of hedonia. Psychopharmacology 2001;157:269-76.
[39] Voikar V, Polus A, Vasar E, Rauvala H. Long-term individual housing in C57BL/6 and DBA/2 mice: assessment of behavioural consequences. Gen Brain Behav 2005;4(4):240-52.

[40] Benaroya-Milshtein N, Hollander N, Apter A, Kukulansky T, Raz N, Wilf A, et al. Environmental enrichment in mice decreases anxiety, attenuated stress responses and enhances natural killer cell activity. Eur J Neurosci 2004;20:1341-7.

[41] Van de Weerd HA, Van Loo PLP, Van Zutphen LFM, Koolhaas JM, Baumans $V$. Nesting material as environmental enrichment has no adverse effects on behaviour and physiology of laboratory mice. Physiol Behav 1997;62: 1019-28.

[42] Kirchner JM, Bloom AJ, Skutnick-Henley P. The relationship between performance anxiety and flow. Med Probl Perform Art 2008;23:59-65.

[43] Clay AW, Bloomsmith MA, Marr MJ, Maple TL. Habituation and desensitization as methods for reducing fearful behaviour in singly housed rhesus macaques. Am J Primatol 2009;71:30-9.

[44] Martin P, Bateson P. Measuring behaviour. 3rd ed. Cambridge University Press; 2007.

[45] Hurlburt S. Pseudoreplication and the design of ecological field experiments. Ecol Monogr 1984;54(2):187-211.

[46] Akre A, Bakken M, Hovland A, Palme R, Mason G. Clustered environmental enrichments induce more aggression and stereotypic behaviour than do dispersed enrichments in female mice. Appl Anim Behav Sci 2011;131: 145-52.

[47] Burn C, Mason G. Rats seem indifferent between their own scent-marked homecages and clean cages. Appl Anim Behav Sci 2008;115:201-10.

[48] van Dellen A, Blakemore C, Deacon R, York D, Hannan A. Delaying the onset of Huntington's in mice. Nature 2000;404:721-2.

[49] Sztainberg Y, Chen A. An environmental enrichment model for mice. Nat Protoc 2010;5:1535-9. 